\title{
Numerical Simulation of Premixed Turbulent Methane Combustion
}

\author{
Marc Day \\ MSDay@Ibl.gov \\ Center for Computational Sciences and Engineering \\ Lawrence Berkeley National Laboratory, USA \\ http://seesar.lbl.gov/ccse/
}
Presented at: Second M.I.T. Conference on Computational Fluid and Solid Mechanics Massachusetts Institute of Technology
Cambridge, MA
June 2003

Collaborators: J. Bell, A. Almgren, V. Beckner, M. Lijewski, R. Cheng, I. Shepherd, M. Johnson 


\section{Objective}

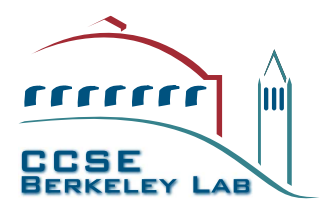

Simulate laboratory-scale turbulent premixed combustion using detailed kinetics and transport without subgrid models for turbulence or turbulence-chemistry interaction

Application: Turbulent laboratory flames

- Fundamental flame dynamics

- Pollutant $\left(\mathrm{NO}_{x}\right)$ formation

Premixed Low-Swirl Burner

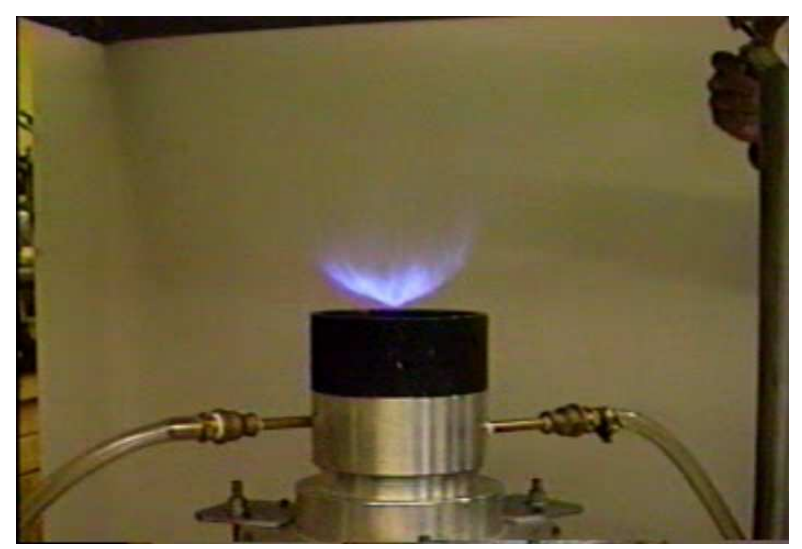

Traditional approach: Compressible DNS

- High-order explicit finite-differences

- At least $O\left(10^{9}\right)$ zones

- At least $O\left(10^{6}\right)$ timesteps

Rod-stabilized Flame

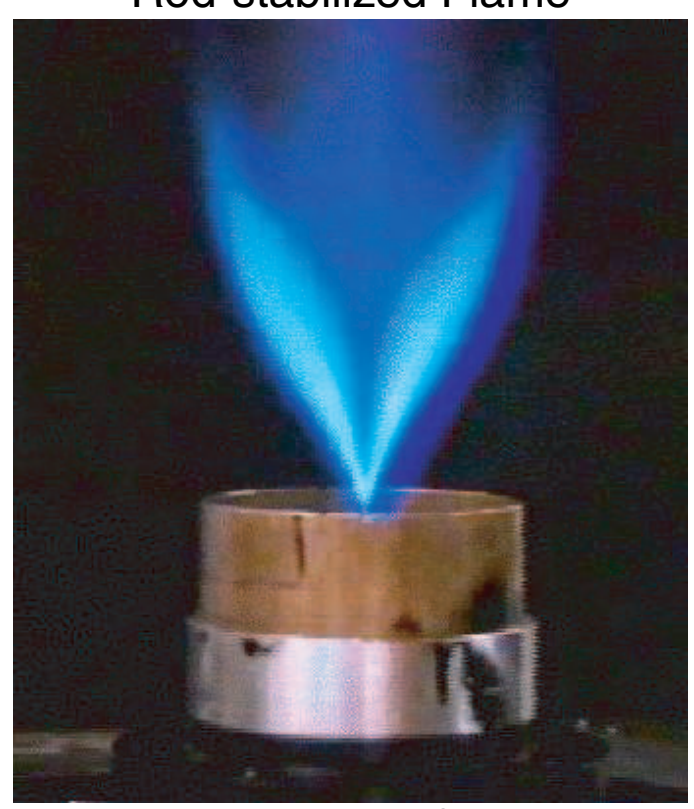

Photo courtesy R. Cheng 


\section{Approach}

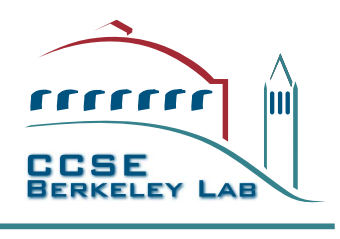

With traditional methods, laboratory-scale simulations with detailed chemistry and transport are intractable for the near future

Observation:

- Laboratory turbulent flames are low Mach number

- Regions requiring high-resolution are localized in space

Our approach:

- Low Mach number formulation

- Eliminate acoustic time-step restriction while retaining compressibility effects due to heat release

- Cost: Linear algebra associated with elliptic constraint

- Adaptive mesh refinement

- Localize mesh where needed

- Cost: Complexity from synchronization of elliptic solves

- Parallel architectures

- Distributed memory implementation using BoxLib framework

- Cost: Dynamic load balancing of heterogeneous work load 


\section{Low Mach Number Combustion}

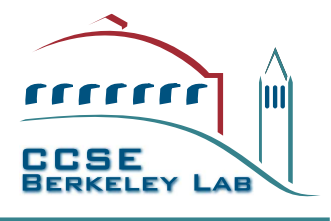

Low Mach number model, $M=U / c \ll 1$ (Rehm \& Baum 1978, Majda \& Sethian 1985)

$$
p(\vec{x}, t)=p_{0}(t)+\pi(\vec{x}, t) \quad \text { where } \quad \pi / p_{0} \sim \mathcal{O}\left(M^{2}\right)
$$

- $p_{0}$ does not affect local dynamics, $\pi$ does not affect thermodynamics

- Acoustic waves analytically removed (or, have been "relaxed" away)

- $\vec{U}$ satisfies a divergence constraint, $\nabla \cdot \vec{U}=S$

Conservation equations:

$$
\begin{aligned}
& \rho \frac{D \vec{U}}{D t}+\nabla \pi=\nabla \cdot \tau \\
& \frac{\partial \rho Y_{\ell}}{\partial t}+\nabla \cdot\left(\rho Y_{\ell} \vec{U}\right)=\nabla \cdot \vec{F}_{\ell}+\rho \dot{\omega}_{\ell} \\
& \frac{\partial \rho h}{\partial t}+\nabla \cdot(\rho h \vec{U})=\nabla \cdot \vec{Q}
\end{aligned}
$$

- $Y_{\ell}$ mass fraction

- $\vec{F}_{\ell}$ species diffusion, $\sum \vec{F}_{\ell}=0$

- $\dot{\omega}_{\ell}$ species production, $\sum \dot{\omega}_{\ell}=0$

- $h$ enthalpy $h=\sum Y_{\ell} h_{\ell}(T)$

- $\vec{Q}$ heat flux

- $p=\rho R T \sum Y_{\ell} / W_{\ell}$ 


\section{Fractional Step Approach}

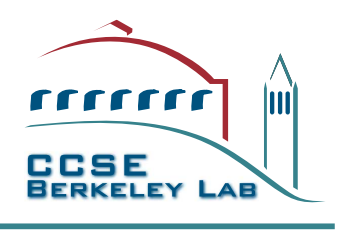

$$
\begin{aligned}
\text { Operator-split Integration: } & \text { - Explicit advection } \\
& \text { - Semi-implicit diffusion } \\
& \text { - Implicit chemistry }
\end{aligned}
$$

Time Advance Summary:

1. Preliminary $U^{*}$ update using lagged $\nabla \pi$, ignore divergence constraint.

2. Update species, enthalpy and temperature. Compute updated $S$.

3. Decompose $U^{*}$ to extract the component satisfying $\nabla \cdot U=S$.

Decomposition achieved by solving a linear elliptic equation for $\phi$

$$
\nabla \cdot\left(\frac{1}{\rho} \nabla \phi\right)=\nabla \cdot U^{*}-S^{n+1}
$$

Final $U$ and $\pi$ update using $\phi$ :

$$
U=U^{*}-\frac{1}{\rho} \nabla \phi \quad \text { and } \quad \pi^{n+1 / 2}=\pi^{n-1 / 2}+\phi
$$




\section{Properties of the methodology}

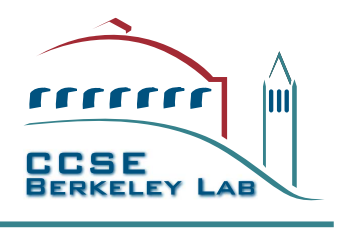

1. Overall formulation is second-order accurate in space and time.

2. Godunov discretization provides robust advective transport.

3. Strictly conserves species, mass and energy.

4. Ideal gas equation of state only approximately satisfied

$$
p_{o} \neq \rho R T \sum_{m} \frac{Y_{m}}{W_{m}}
$$

Modified divergence constraint minimizes drift from EOS 
Block-structured hierarchical grids

Each grid patch (2D or 3D)

- Logically structured, rectangular

- Refined in space and time by evenly dividing coarse grid cells

- Dynamically created/destroyed to track time-dependent features

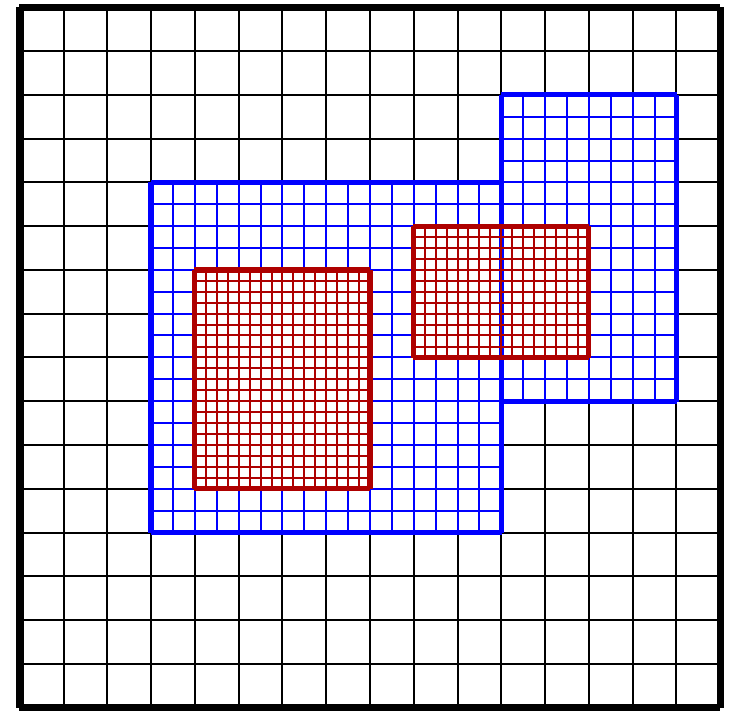

2D adaptive grid hierarchy

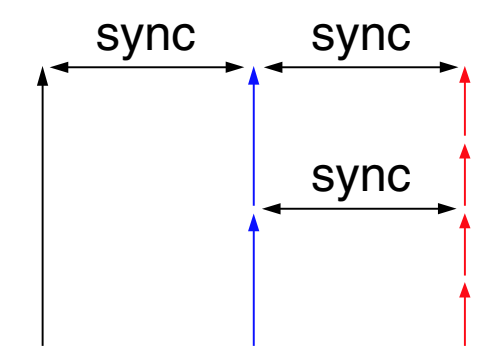

Level 0 Level $1 \quad$ Level 2

- Synchronize levels $\ell$ and $\ell+1$

\section{Preserves properties of single-grid algorithm}




\section{AMR Level Operations}

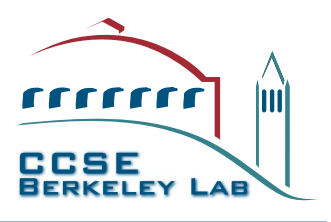

Organize grids by refinement level, couple through "ghost" cells

$\square$ Fine-Fine

- Physical BC

$\square$ Coarse-Fine

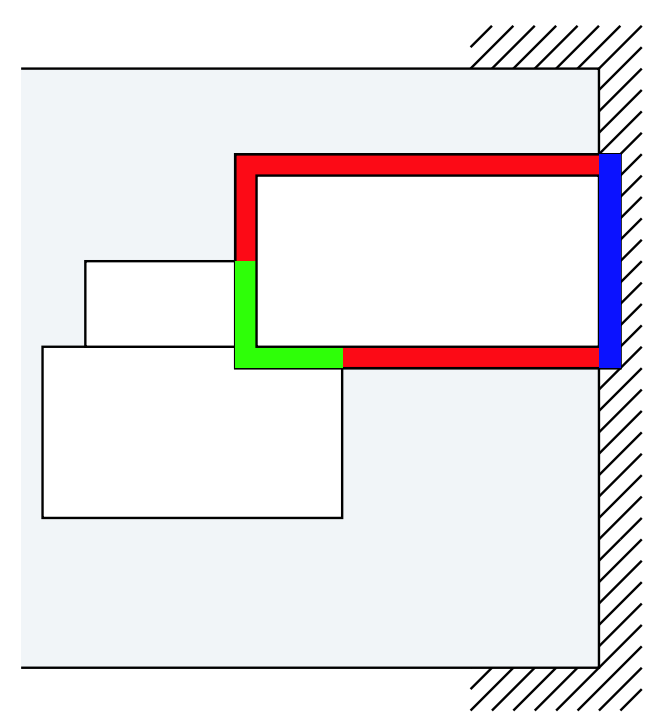

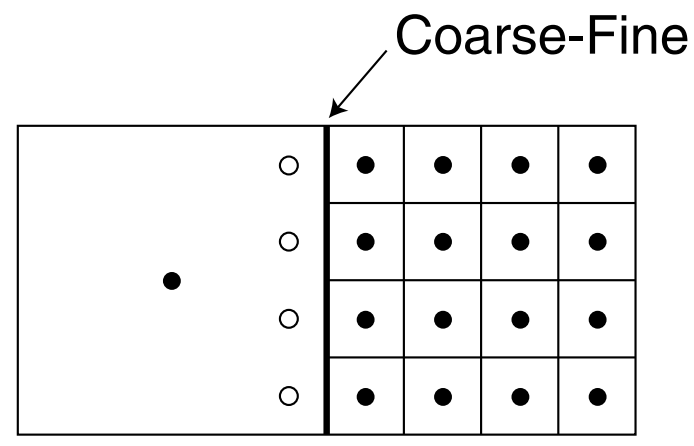

- Level data

- Interpolated data

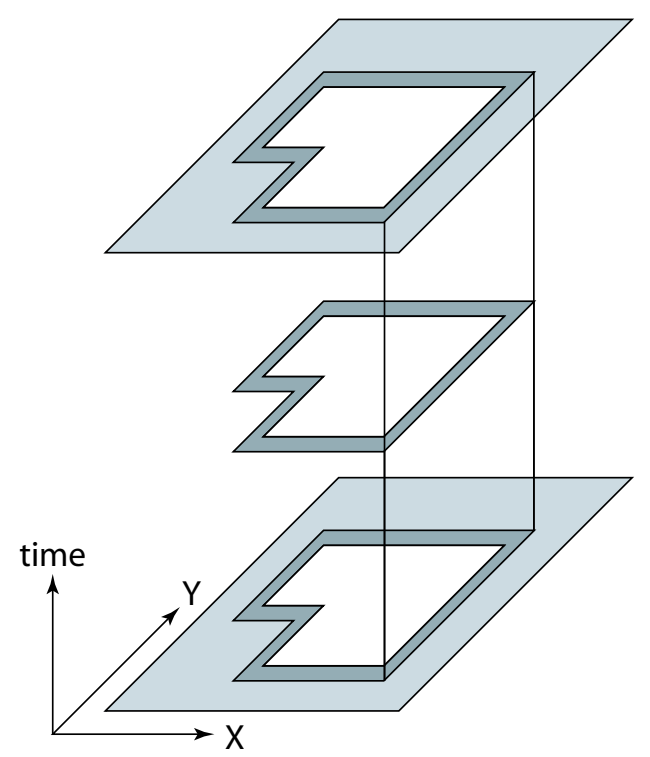




\section{Dynamic Load-Balancing}

Approach: Estimate work per grid, distribute using heuristic KNAPSACK algorithm

Cells/grid often a good work estimate, but chemical kinetics may be highly variable

- Monitor chemistry integration work

- Distribute chemistry work based on this work estimate

Parallel Communication: AMR data communication patterns are complex

- Easy: distribute grids at a single level, minimize off-processor communication

- Hard: Incorporate coarse-fine interpolation (also, "recursive" interpolation)

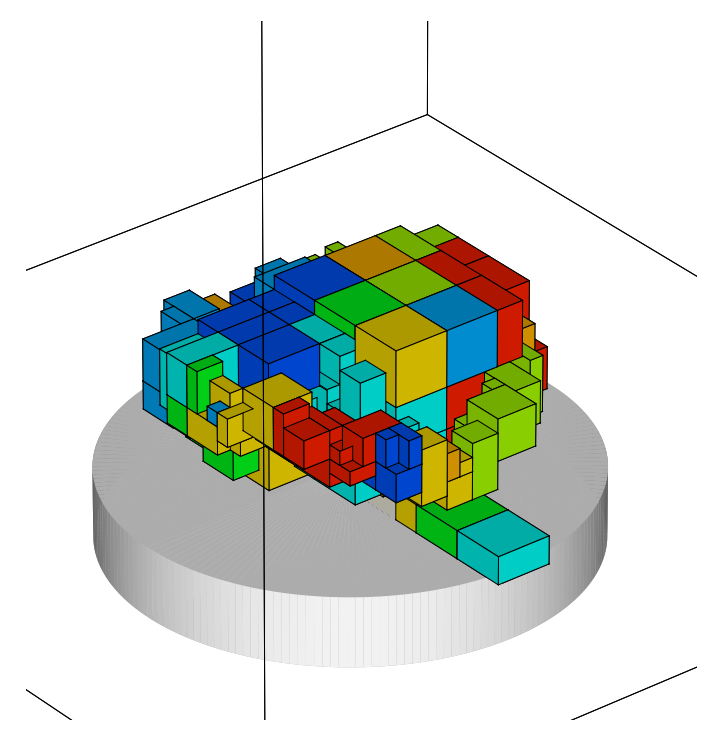

Level 2

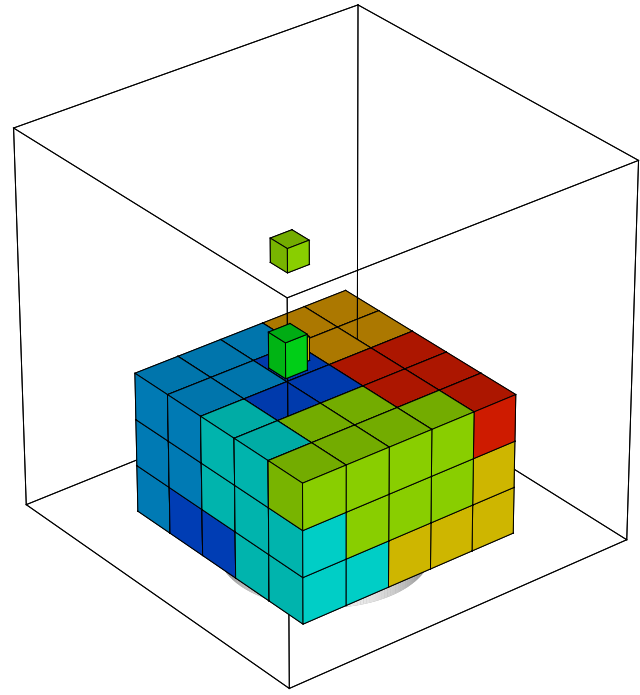

Level 1

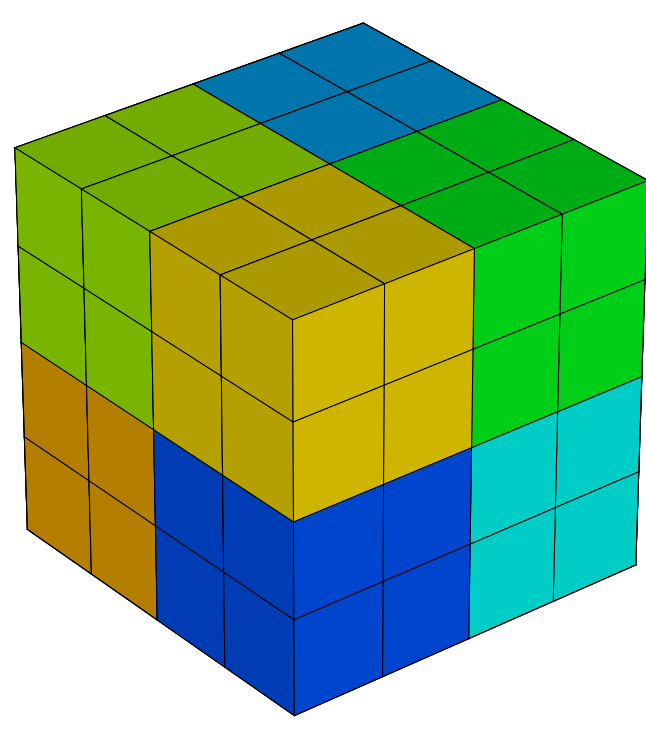

Level 0 


\section{Full-Scale Simulations}

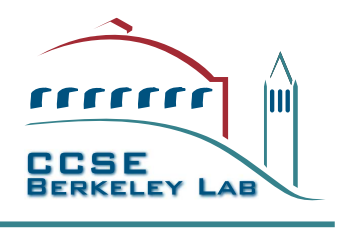

\section{Strategy: Use separate nonreacting (in)compressible simulations to characterize flow into domain from nozzle}

Nozzle simulations:

- For swirl burner, compressible effects important $\left(U_{\max } \sim 0.4 C_{s}\right)$

- For V-flame, all flow is low speed, use incompressible model

- Create inflow field for 3D reacting low Mach number model

- Shaped synthetic turbulence or

- Direct data input

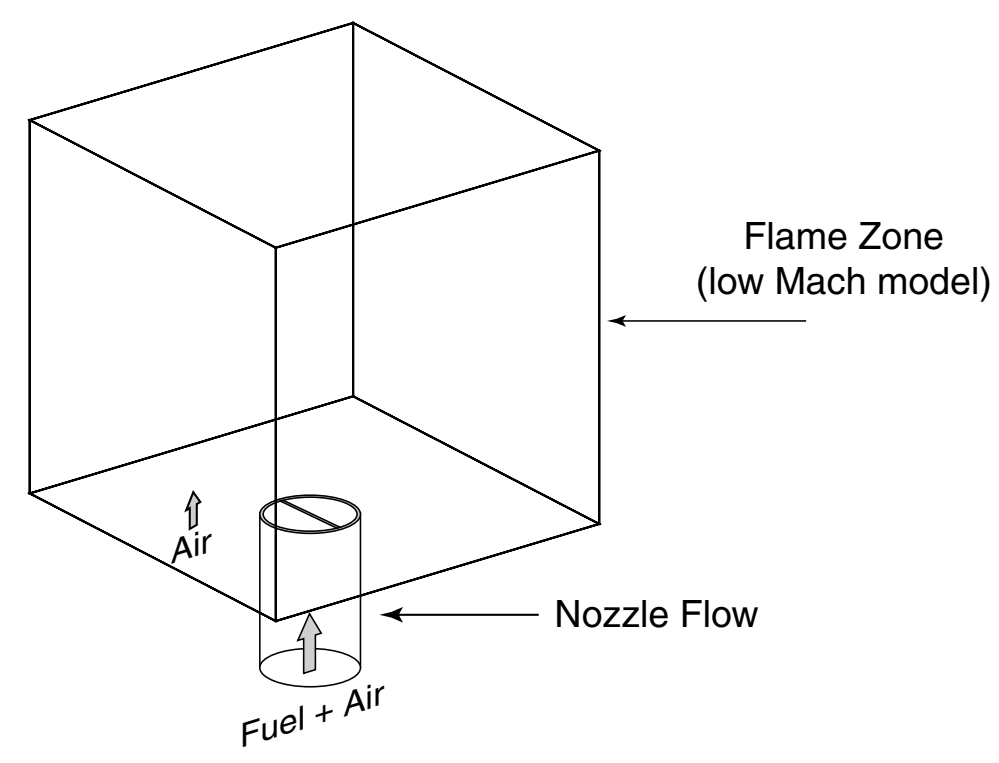




\section{Laboratory-Scale Application}

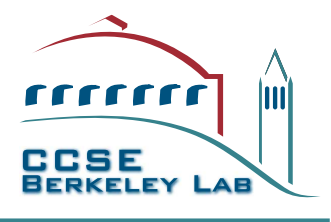

LBNL EETD laboratory turbulent premixed methane flames (In collaboration with R. Cheng, I. Shepherd and M. Johnson)

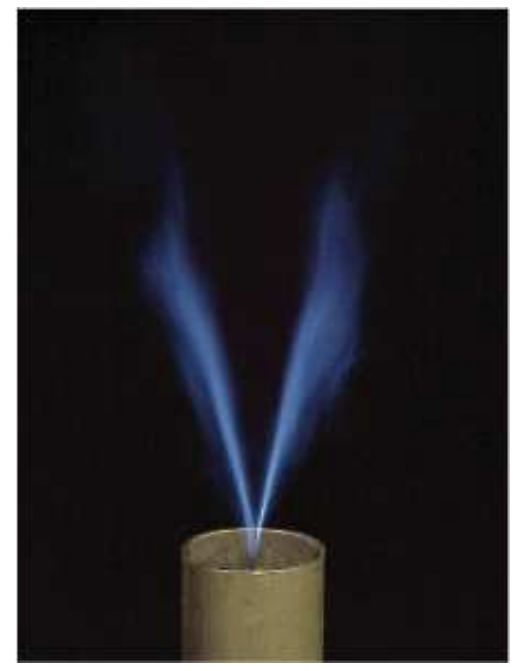

Rod-stabilized V-flame

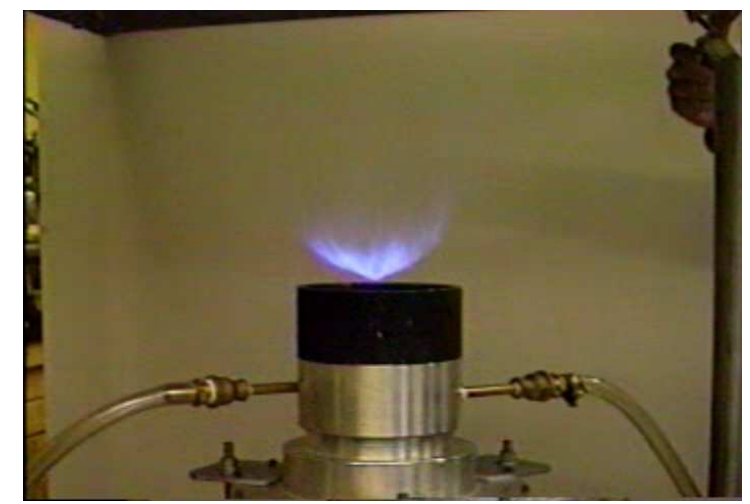

Low-swirl burner

Common Features: Large equivalent turbulent flame speed. (Presumably due to highly wrinkled flame)

Diagnostics: P.I.V. images give instantaneous planar flame shape and 2D velocity map 


\section{Configuration}

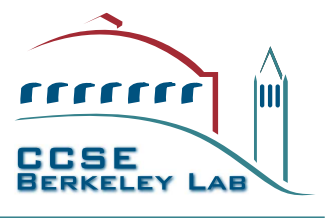

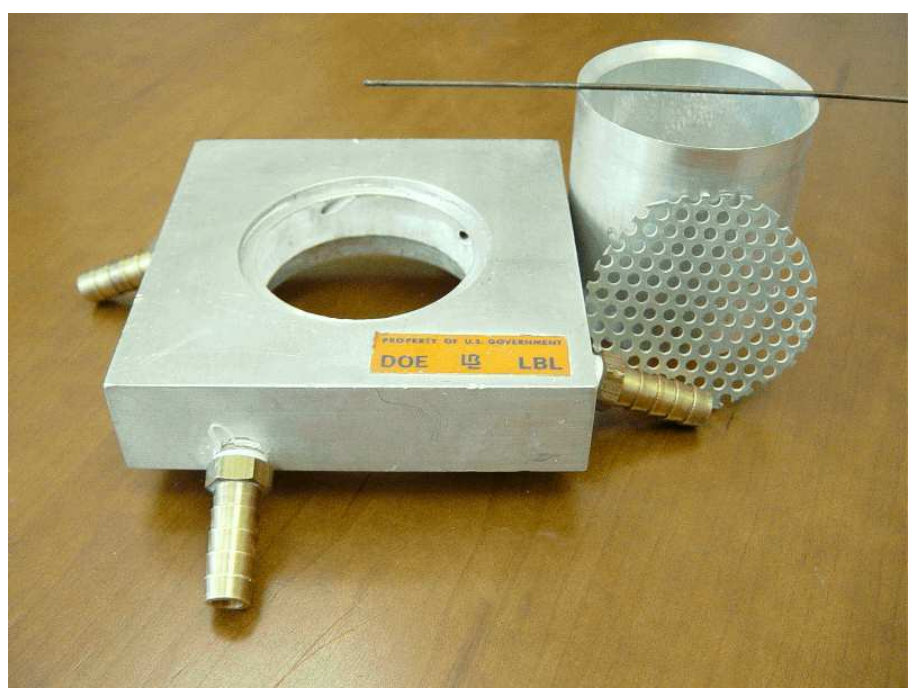

Burner assembly

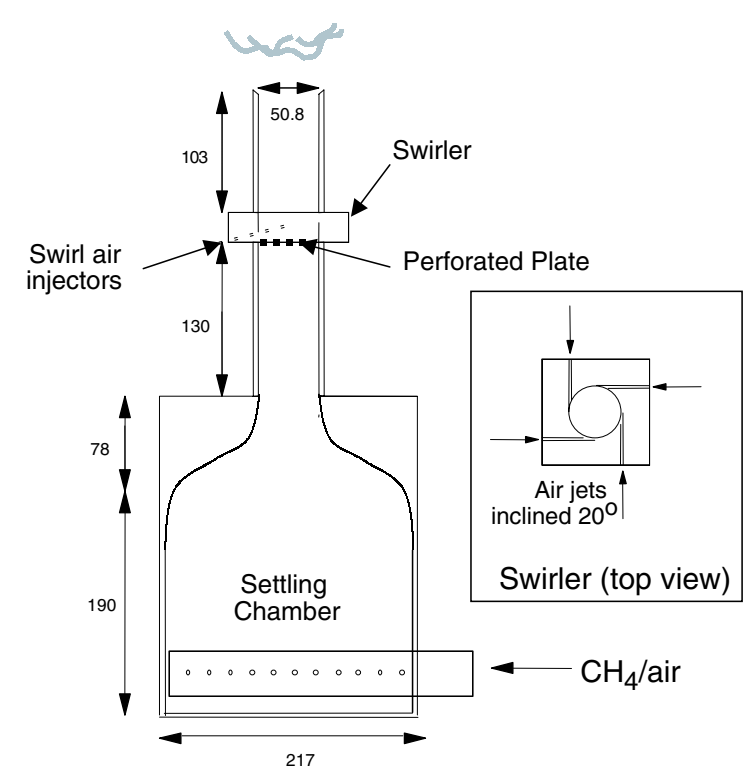

Experiment schematic

- Tangential air jets: $\dot{m}_{a i r} / \dot{m}_{f u e l} \sim .5 / 12.5$

(Swirl number $S \sim 1.16$ )

- V-flame $\left(\dot{m}_{a i r} \equiv 0\right):$ rod $\sim 1 \mathrm{~mm}$

- Turbulence plate: $3 \mathrm{~mm}$ holes on $5 \mathrm{~mm}$ center generates $\ell_{t} \sim 3.5 \mathrm{~mm}, u^{\prime} \sim 0.18 \mathrm{~m} / \mathrm{s}$ 


\section{V-flame Nozzle Flow}

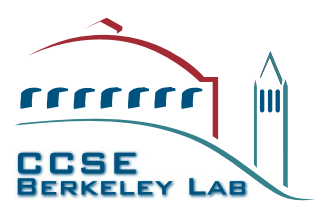

Observe: Within nozzle turbulence plate minimizes boundary effects

Suggests: Fluid evolution across nozzle equivalent to boundary-free Lagrangian evolution over mean nozzle transit period.

Procedure: Incompressible model, triply-periodic domain. Initially opposed jets represent flow through plate holes. Evolve for $t=L / \bar{U}$.

Results: $\ell_{t}$ and $u^{\prime}$ consistent with experimental observation

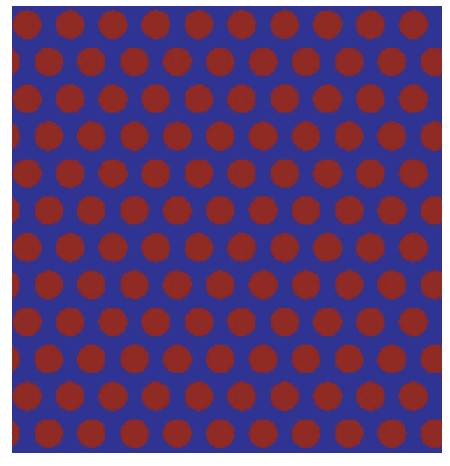

Initial $u_{z}(-3,+4.5) \mathrm{m} / \mathrm{s}$ - zero net flow

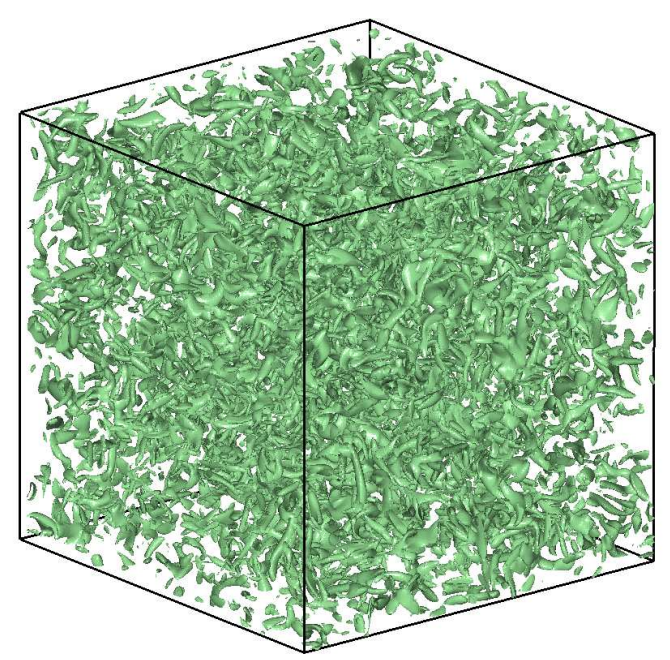

Simulated vorticity, $t=.03 \mathrm{sec}$.

Shape resulting field to $u^{\prime} \rightarrow 0$ as $r \rightarrow R_{f}$ (and over rod), flow into bottom. 
- DRM-19 methane mechanism (20 species, 84 reactions)

- Species-dependent mixture-averaged transport

- Initialize premixed flame near rod, evolve until quasi-steady

- Adapt grid to track flame surface (HCO) and high vorticity

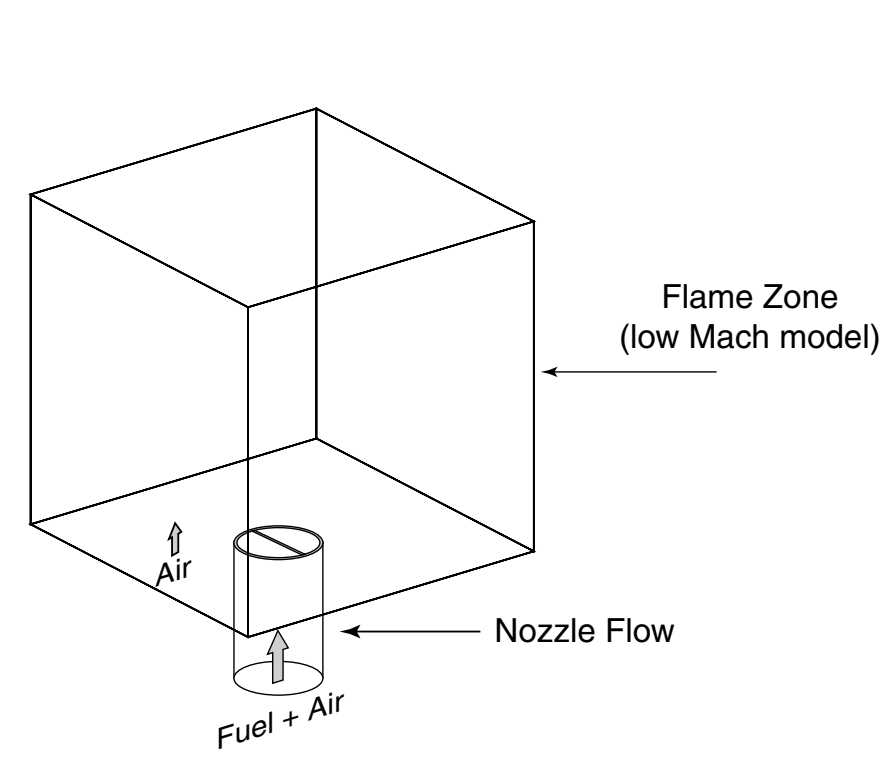

Computational domain $(12 \mathrm{~cm})^{3}$

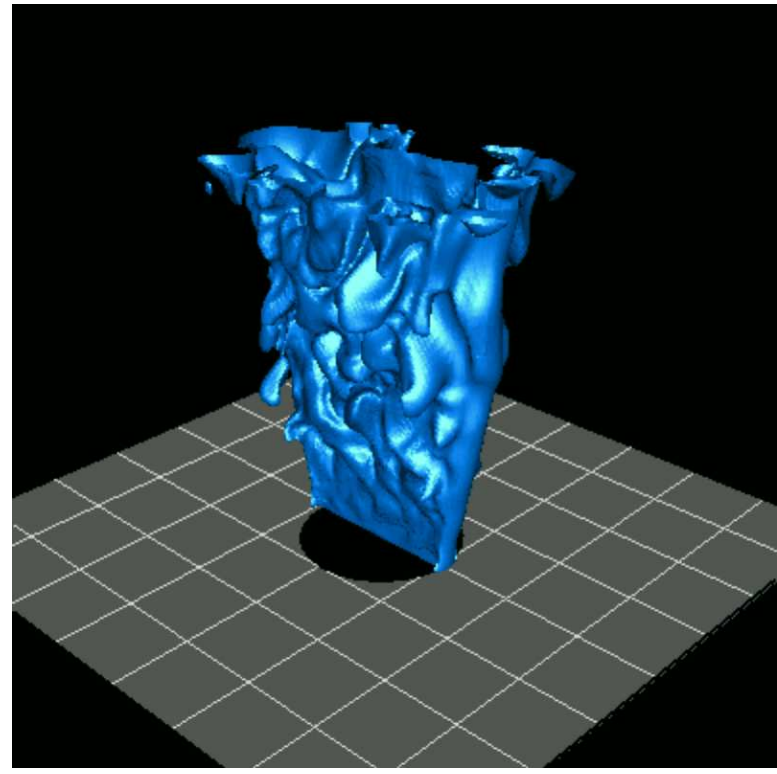

Quasi-steady simulated V-flame

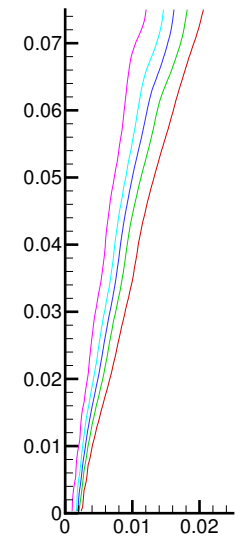

$\bar{c}$ (progress variable)

Total simulation time $=.136 \mathrm{sec}$ (3.5 times thru domain at $3 \mathrm{~m} / \mathrm{s}$ ) $\Delta x_{\text {finest }}=117 \mu \mathrm{m}$ over $15 \%$ of domain 


\section{V-flame Validation - Work-In-Progress}

Instantaneous flame location

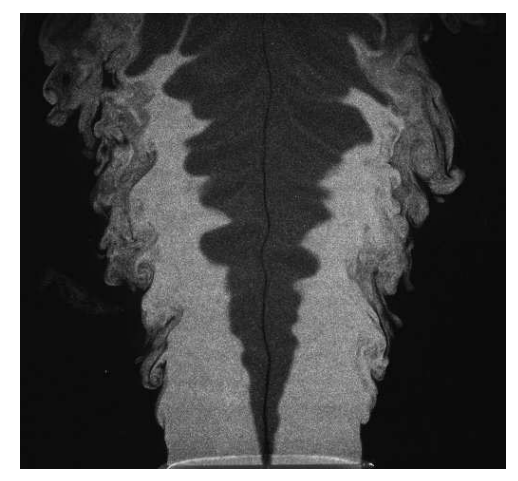

Expt: PIV image

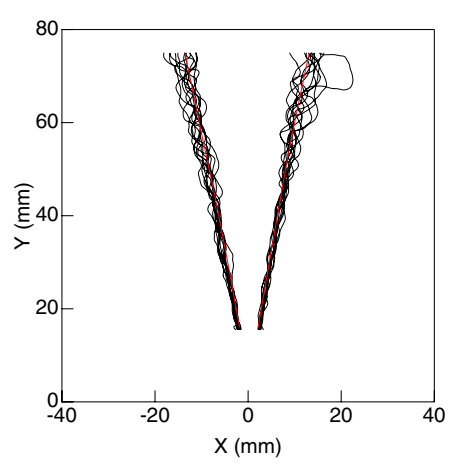

Expt: Vertical cuts

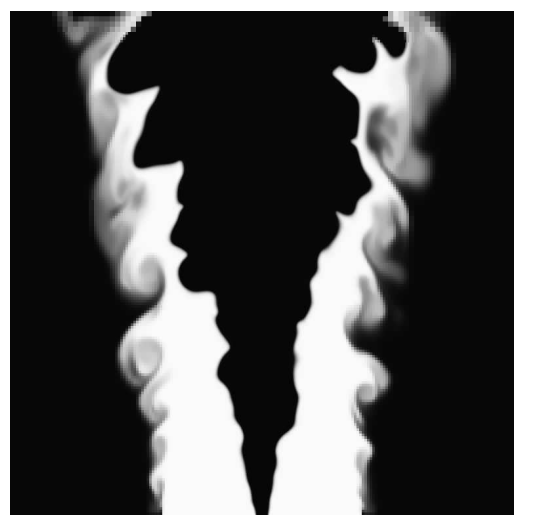

Simulation: $\mathrm{X}\left(\mathrm{CH}_{4}\right)$

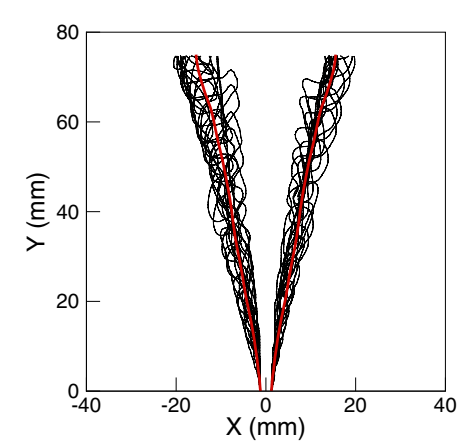

Simulation: Vertical cuts
Observe:

- Good qualitative agreement

- Features invariant to $2 x$ grid resolution $(\Delta x=59 \mu \mathrm{m})$

- Turbulent flame speed $\left(\dot{\omega}_{C H 4}\right)$ enhancement $S_{t}=1.9 S_{L}$

- Area enhancement due to wrinkling $A_{t}=1.25 A_{L}$

In Progress:

- Quantitative validations

- 2D vs. 3D flame stats

- Turb/chem interaction analysis using $59 \mu \mathrm{m}$ data 


\section{Low-Swirl Simulations - Inlet}

Observation: Earlier scheme invalid since compressibility/wall effects significant with air jets $\sim 40 \%$ sound speed.

Levels of Simulation Detail:

1. Synthetic turbulence (isotropic/decaying), with "tophat" shaping, combined with axisymmetric guess for swirl/fuel profiles

2. Synthetic turbulence with mean and fluctuating components derived from a full, compressible nozzle simulation

$\Longrightarrow$ 3. Coupled solution with full 3D time-dependent inflow boundary data 


\section{Compressible Flow with Geometry}

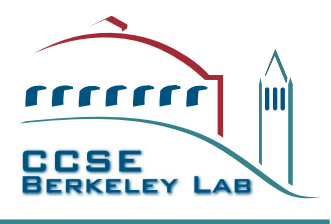

Model geometry as front embedded in regular Cartesian grid

- Volume fractions

- Area Fractions

Finite volume discretization (Chern and Colella)

- Conservative update unstable in small cells

- Update with stable fraction

- Distribute remainder to neighboring cells

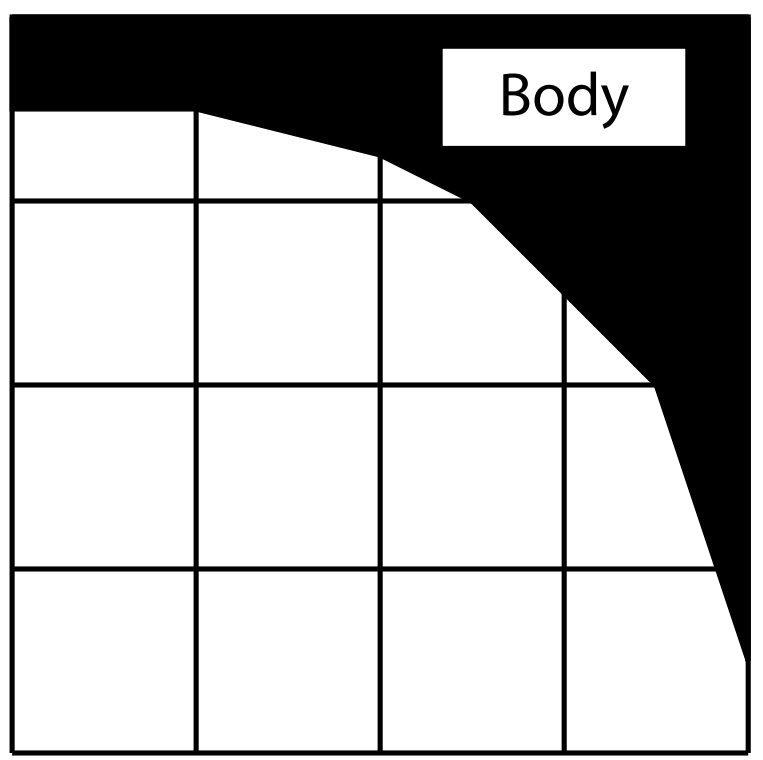

Adaptive, parallel, 3D, ...

Pember et al., JCP, 1995 


\section{Nozzle Geometry}

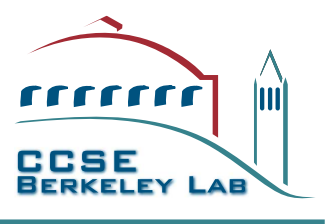

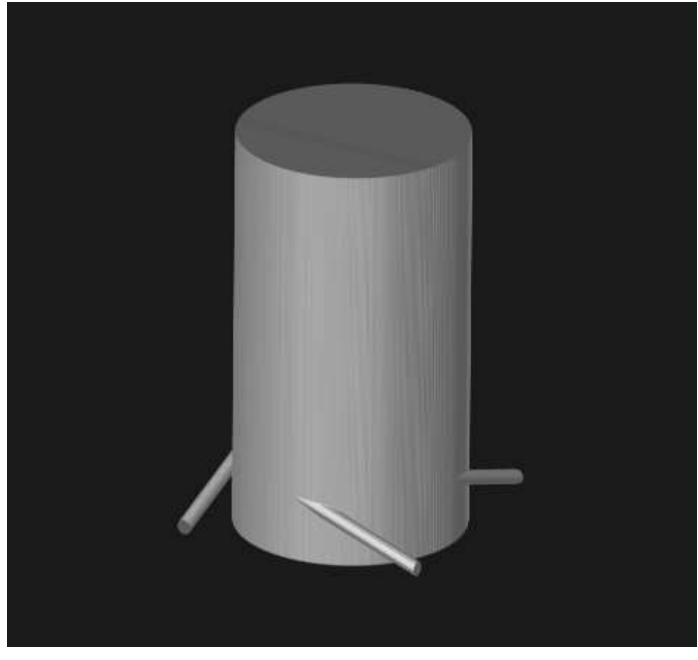

Flow domain for swirl nozzle

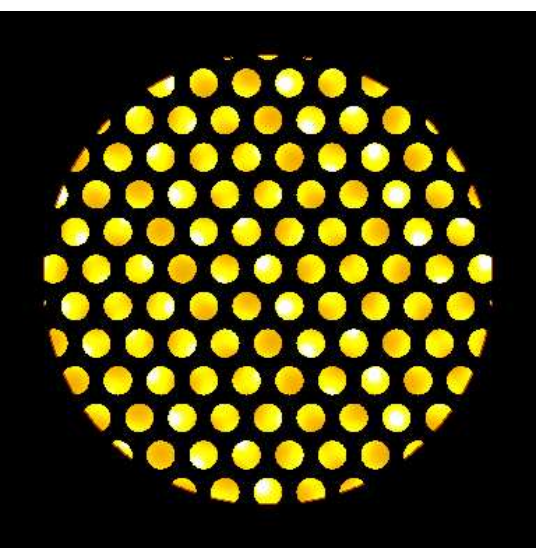

Turbulence plate for nozzle inlet
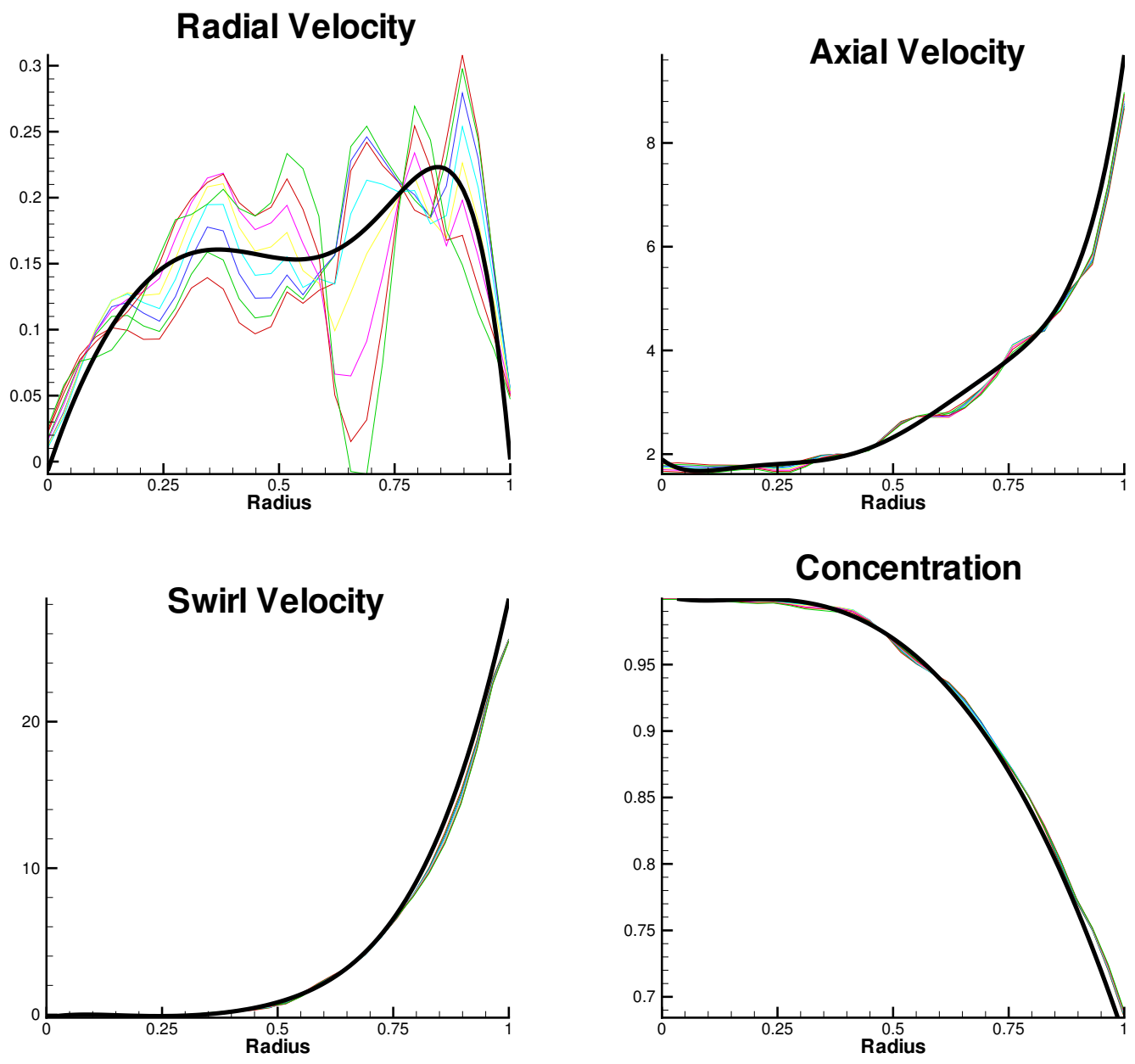

Simulated mean profiles 


\section{Swirling Nozzle Flow}

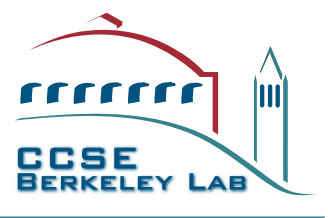

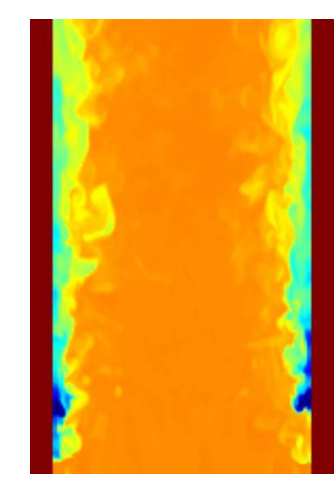

Fuel (orange) and air (blue) inside nozzle

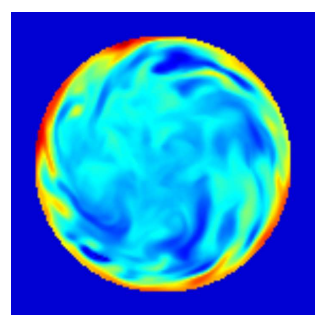

Axial velocity at nozzle exit
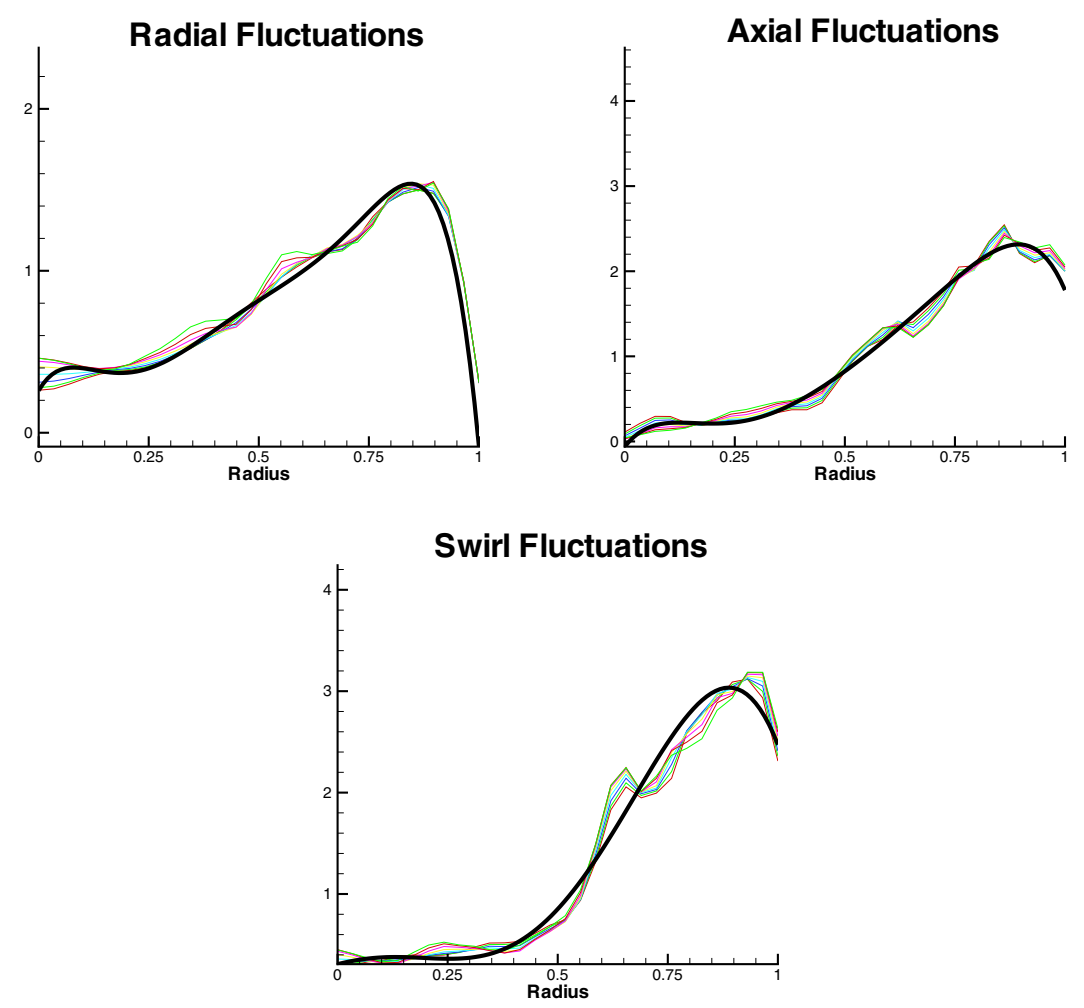

Fluctuation profiles from compressible simulation

Observe: Significant radial fluctuations Large $u_{z}, u_{\theta}$ in air boundary layer Considerable azimuthal activity 


\section{Low Swirl Burner - Preliminary Results}
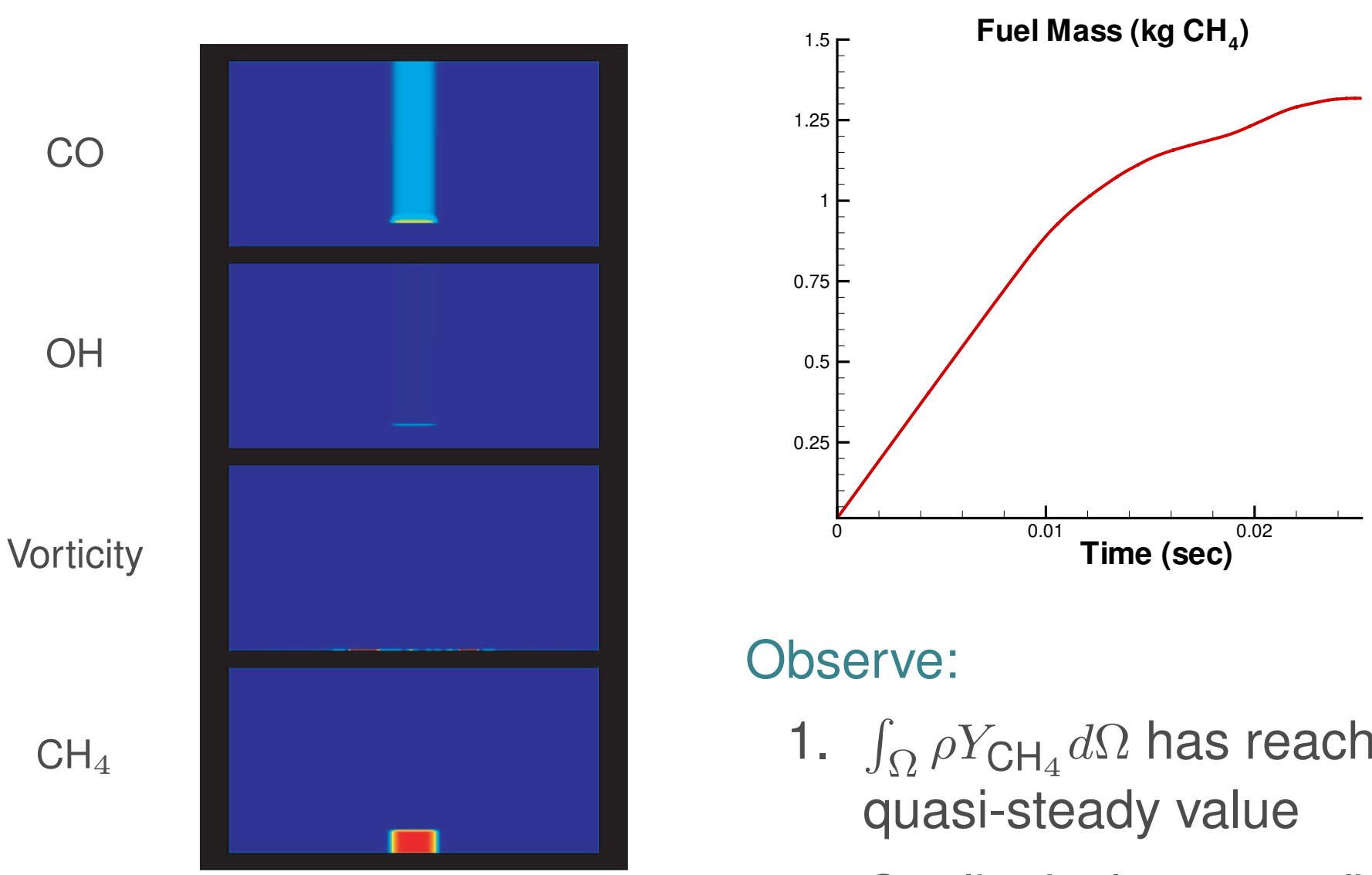

Observe:

1. $\int_{\Omega} \rho Y_{\mathrm{CH}_{4}} d \Omega$ has reached quasi-steady value

2. Qualitatively correct flame, flow field shape 


\section{Summary and Future Work}

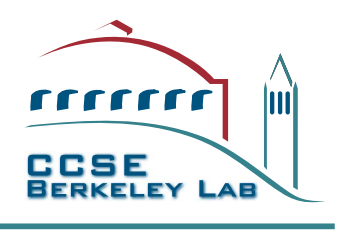

Algorithm for low Mach number combustion

- Adaptive

- Conservative

- Second-order in time and space

- Parallel

Application to laboratory-scale turbulent premixed combustion

- Rod-stabilized V-flame

- Low-swirl burner

- Auxiliary compressible/incompressible simulations provide inlet boundary data from turbulent nozzle

Future Work

- Futher validations

- Quantitative comparison with experiment

- Characterize turbulent flame propagation properties

- Investigate turbulent flame chemistry 